\title{
Frequency and risk factors for postoperative aneurysm residual after microsurgical clipping
}

\author{
Kathrin Obermueller ${ }^{1}$ (1) $\cdot$ Isabel Hostettler ${ }^{1} \cdot$ Arthur Wagner $^{1} \cdot$ Tobias Boeckh-Behrens $^{2} \cdot$ Claus Zimmer $^{2}$. \\ Jens Gempt ${ }^{1}$ • Bernhard Meyer ${ }^{1}$. Maria Wostrack ${ }^{1}$
}

Received: 17 July 2020 / Accepted: 27 October 2020 / Published online: 20 November 2020

(C) The Author(s) 2020

\begin{abstract}
Objective Aneurysm residuals after clipping are a well-known problem, but the course of aneurysm remnants in follow-up is not well studied. No standards or follow-up guidelines exist for treatment of aneurysm remnants. The aim of this study was to evaluate the risk factors for postoperative aneurysm remnants and their changes during follow-up.

Methods We performed a retrospective analysis of 666 aneurysms treated via clipping in our hospital from 2006 to 2016. Postoperative and follow-up angiographic data were analyzed for aneurysm remnants and regrowth. Clinical parameters and aneurysm-specific characteristics were correlated with radiological results.

Results The frequency of aneurysm residuals was $12 \%$ (78/666). Aneurysms located in the middle cerebral artery $(p=0.02)$ showed a significantly lower risk for incomplete aneurysm occlusion. Larger aneurysms with a diameter of 11-25 mm $(p=$ 0.005 ) showed a significantly higher risk for incomplete aneurysm occlusion. Five patients underwent re-clipping during the same hospital stay. Remnants were stratified based on morphological characteristics into "dog ears" $(n=60)$ and "broad based" $(n=13)$. The majority of the "dog ears" stayed stable, decreased in size, or vanished during follow-up. Broad-based remnants showed a higher risk of regrowth.

Conclusions A middle cerebral artery location seems to lower the risk for the incomplete clip occlusion of an aneurysm. Greater aneurysm size $(11-25 \mathrm{~mm})$ is associated with a postoperative aneurysm remnant. The majority of "dog-ear" remnants appear to remain stable during follow-up. In these cases, unnecessarily frequent angiographic checks could be avoided. By contrast, broadbased residuals show a higher risk of regrowth that requires close imaging controls if retreatment cannot be performed immediately.
\end{abstract}

Keywords Intracranial aneurysm $\cdot$ Aneurysm clipping $\cdot$ Aneurysm residual $\cdot$ Aneurysm remnant $\cdot$ Aneurysm regrowth

\begin{tabular}{|c|c|c|}
\hline \multirow{2}{*}{\multicolumn{3}{|c|}{ Abbreviations }} \\
\hline & & \\
\hline \multicolumn{2}{|c|}{$\mathrm{ACA}$} & Anterior cerebral artery \\
\hline \multicolumn{2}{|c|}{ DSA } & Digital subtraction angiography \\
\hline \multicolumn{2}{|c|}{ Fig. } & Figure \\
\hline \multicolumn{2}{|c|}{ FU } & Follow-up \\
\hline \multicolumn{2}{|c|}{$\mathrm{H} \& \mathrm{H}$} & Hunt and Hess grade \\
\hline \multicolumn{3}{|c|}{$\begin{array}{l}\text { This article is part of the Topical Collection on Vascular Neurosurgery - } \\
\text { Aneurysm }\end{array}$} \\
\hline$\triangle$ & \multicolumn{2}{|c|}{$\begin{array}{l}\text { Kathrin Obermueller } \\
\text { kathrin.obermueller@tum.de }\end{array}$} \\
\hline \multicolumn{3}{|c|}{$\begin{array}{l}\text { Department of Neurosurgery, Klinikum rechts der Isar, Technical } \\
\text { University Munich, Ismaninger Straße 22, } 81675 \text { Munich, Germany }\end{array}$} \\
\hline 2 & $\begin{array}{l}\text { Department o } \\
\text { University M }\end{array}$ & $\begin{array}{l}\text { roradiology, Klinikum rechts der Isar, Technical } \\
\text { Ismaninger Straße 22, } 81675 \text { Munich, Germany }\end{array}$ \\
\hline
\end{tabular}

$\begin{array}{ll}\text { ICA } & \begin{array}{l}\text { Internal carotid artery } \\ \text { ICD }\end{array} \\ \text { ICG angiography } \\ \text { of Diseases and Related Health Problems } \\ \text { Indocyanine green angiography } \\ \text { MCA } & \text { Middle cerebral artery } \\ \text { PC } & \text { Posterior circulation } \\ \text { SAH } & \text { Subarachnoid hemorrhage } \\ \text { SD } & \text { Standard deviation } \\ \text { Tab. } & \text { Table }\end{array}$

Introduction

The frequency of incomplete aneurysm occlusion after surgical clipping varies from $2-49 \%$ in different surgical series $[1-5,7,9,12,13,15,20,24,25,27,28,31,32]$. The risk of 
rebleeding after aneurysm clipping is estimated at $1.3 \%$ and is associated with the size of the residual rest [19]. However clear criteria for retreatment and for follow-up controls of aneurysm residuals have not yet been strictly defined. Possible risk factors and potential follow-up dynamics are essential for treatment decisions, as well as for patient consultation.

Postoperative angiography is an accepted standard in most neurosurgical departments, as it reveals residual filling of aneurysms or other complications, like major vessel occlusion $[13,20,27,28]$. In patients with completely clipped aneurysms, further angiographic controls for the treated aneurysms are considered unnecessary. However, no standards have been established for follow-up (FU) in cases with residual aneurysms after clipping. Currently, aneurysm remnants are categorized according to their morphological characteristics. David et al. and Raymond et al. classified aneurysm remnants into 2 categories: "dog-ear" and "broad-based" residuals. "Dog ears" consist of a small neck remnant between the parent vessel and the base of the clip, whereas the reconstructed parent vessel of "broad-based" remnants contains part of the aneurysm wall [9, 29]. These differences in morphology suggest different risks for regrowth and subsequent rebleeding. Accordingly, the follow-up periods and the decision for surgery should be made based on the estimated risk of rupture.

The aim of this study was to determine the frequency and risk factors of residual aneurysm filling after aneurysm clipping and to estimate the risk of regrowth of remnants in our surgical series.

\section{Methods}

We performed a retrospective, single-center data analysis of patients treated for intracranial aneurysm via clipping. The analyzed data were collected from 2006 until 2016.

\section{Patient inclusion/exclusion criteria}

Based on the operational key for aneurysm clipping, the patient data were consecutively entered into the database. We included patients with clipping for incidental aneurysms and patients who were clipped for ruptured aneurysms. Patients who received surgical treatment other than clipping (e.g., wrapping or trapping and bypass surgery) or, who did not undergo postoperative digital subtraction angiography (DSA) were excluded from the final analysis. Patients who underwent aneurysm clipping in the context of other pathologies (for example, arteriovenous malformation, or mycotic aneurysms) were also excluded from the analysis. The local ethics committees approved the study protocol (nb. 5020/11)

\section{Interventions}

Every patient received a four-vessel catheter DSA before and a target vessel DSA within the first $24 \mathrm{~h}$ after surgery. The decision for the treatment modality was based on interdisciplinary discussion between the neuroradiologist and neurosurgeon. All surgeries were performed by three experienced vascular neurosurgeons. For all incidental aneurysms, and partly for ruptured aneurysms, patients underwent intraoperative neuromonitoring comprising trans-cranially recorded motor evoked potentials. Indocyanine green (ICG) angiography was routinely conducted. If any aneurysm residual or vessel occlusion was suspected in postoperative angiography, the findings were discussed immediately to provide the possibility of a prompt clip adjustment. Indications for FU angiography in cases of residual filling without an indication for clip repositioning were discussed interdisciplinarily and were performed depending on the size, shape, and general condition of the patient.

\section{Outcomes}

The following clinical, radiological, and epidemiological data were recorded for each patient: age, sex, preoperative neurological condition, Hunt and Hess grade, Fisher grade, aneurysm location and size, number of aneurysms, number of operations, postoperative neurological condition according to the modified Rankin Scale, size of aneurysm remnant, size of aneurysm remnant in FU angiography, and neurological status during FU.

For the present research, postoperative DSA results were screened for aneurysm remnants. An aneurysm remnant was defined as an inflow of contrast medium with a minimum size of $1 \mathrm{~mm}$ and visible on at least 2 projections of angiography in the area of a previously described aneurysm. Follow-up examinations were reviewed for growth of the aneurysm remnant, defined as any new inflow of contrast medium (compared to postoperative angiography) that caused an enlargement of the residual. Changes in flow patterns or thrombosis of the remnant were also evaluated.

Aneurysm remnants were divided according to classification of David et al. and Raymond et al. into "dog-ear" remnants with a small residual between the parent vessel and the base of the clip and "broad-based" remnants, in which a larger residual was still filling [9, 29]. The "dog-ear remnant" group was scheduled for FU angiography depending on the remnant size, the history of any previous subarachnoid hemorrhage $(\mathrm{SAH})$, and the general condition and age of the patient. Patients with "broad-based remnants" were either reoperated during the same hospital stay or were designated for FU imaging. FU DSA examinations were scheduled depending on the individual risk factors and remnant characteristics regularly within a period of 3 months to 1 year after 
surgery. The mean FU in aneurysm remnants was 31 months (SD: \pm 23.3 , range 2.3-93 months).

\section{Statistical analysis}

The study sample was described using means \pm standard deviations for the continuous variables, while categorical parameters were depicted using absolute and relative frequencies. Chi-squared tests and binary logistic regression were used to test categorical variables. Continuous variables were tested with a $t$ test or Wilcoxon test. A $p$ value of 0.05 was considered statistically significant. Analyses were performed utilizing R Studio Version 1.0.4 (R Studio, Boston, USA).

\section{Results}

\section{Data collection}

In 492 patients, a total of 666 ruptured and unruptured aneurysms were treated between April 2006 and December 2016. The reasons for case exclusion are listed in Fig. 1.

In 18 patients, no postoperative DSA was performed. In this group, 11 cases had suffered a severe SAH and had died soon after aneurysm clipping. In 3 cases, other medical circumstances (malignant disease) were treated immediately and were judged more important than the aneurysm. Complications during the initial angiography, with arterial dissection in 2 and symptomatic arterial emboli in 1 case, led to no postoperative DSA. One patient rejected postoperative DSA after elective clipping.

\section{Patient data}

Patient data and data on the treated aneurysms are shown in Tables 1 and 2.

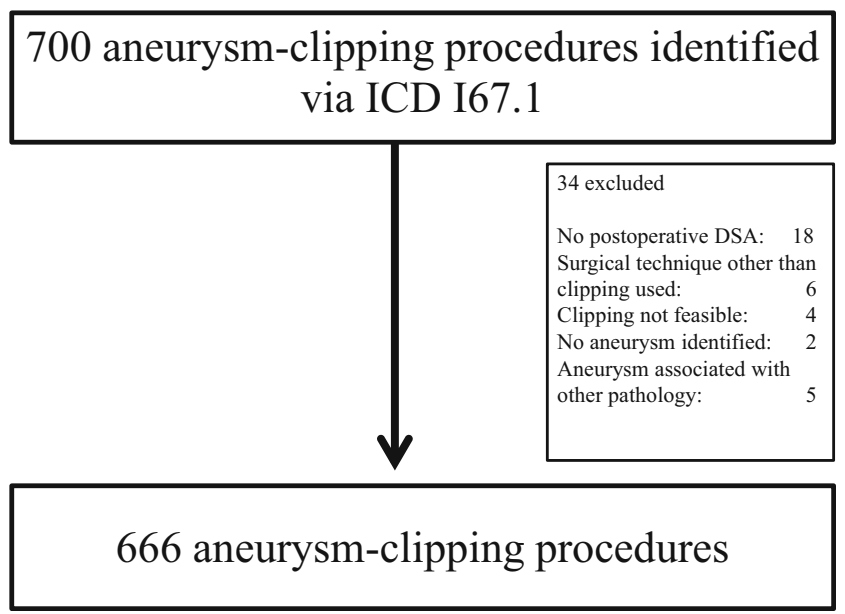

Fig. 1 Flowchart of procedure inclusion via ICD code I67.1
Table 1 Patient data and clinical characteristics $(n=492)$

\begin{tabular}{ll}
\hline Parameter & Value \\
\hline Age at surgery mean \pm SD & $55.48 \pm 13.1$ \\
Gender female $n,(\%)$ & $343(70)$ \\
Acute SAH $n,(\%)$ & $252(51)$ \\
Multiple aneurysms $\geq 2 n,(\%)$ & $167(34)$ \\
\hline
\end{tabular}

$S A H$, subarachnoid hemorrhage

\section{Frequency of postoperative aneurysm remnants}

Residual filling was found in 78/666 aneurysms (12\%). Data on patients with postoperative aneurysm residuals are presented in Table 3.

\section{Risk of aneurysm residuals}

Binary logistic regression revealed a higher risk of an aneurysm residual for aneurysms sized $11-25 \mathrm{~mm}$, with an odds ratio of 9.36 (CI 1.96-44.61; $p=0.005$ ). A location in the MCA showed a significantly lower risk for aneurysm residuals when compared with other locations, with an odds ratio of 0.31 (CI: $0.12-0.83 ; p=0.02$ ) (Table 4). Sex, number of aneurysms per patient, and emergency setting of surgery showed no significant association with postoperative aneurysm residuals.

\section{Follow-up of aneurysm remnants}

Figure 2 shows the classification and early treatment of aneurysm remnants. FU was available for 33/60 (55\%) aneurysms in the dog-ear group and for 7/13 (54\%) in the group of broad-

Table 2 Data on treated aneurysms $(n=666)$

\begin{tabular}{ll}
\hline Parameter & Value \\
\hline Aneurysm location $n,(\%)$ & \\
ACA & $152(23)$ \\
ICA & $140(21)$ \\
MCA & $340(51)$ \\
PC & $34(5)$ \\
Aneurysm size $n,(\%)$ & \\
$<3 \mathrm{~mm}$ & $136(20.4)$ \\
$3-6 \mathrm{~mm}$ & $306(46.0)$ \\
$7-10 \mathrm{~mm}$ & $129(19.4)$ \\
$11-25 \mathrm{~mm}$ & $59(8.8)$ \\
$>25 \mathrm{~mm}$ & $4(0.6)$ \\
Residual of coiled aneurysm & $32(4.8)$ \\
\hline
\end{tabular}

$A C A$, anterior cerebral artery; $I C A$, interior carotid artery; $M C A$, middle cerebral artery; $P C$, posterior circulation 
Table 3 Data on aneurysms with residual filling in postoperative DSA $(n=78)$. The percentage is based on the total number of operated patients in the respective subgroup

\begin{tabular}{ll}
\hline Parameter & Value \\
\hline Aneurysm location & $n,(\%$ of the entire subgroup $)$ \\
ACA $(n=152)$ & $18(11)$ \\
ICA $(n=140)$ & $28(20)$ \\
MCA $(n=340)$ & $25(7)$ \\
PC $(n=34)$ & $8(24)$ \\
Aneurysm size $n,(\%)$ & \\
$<3$ mm $(n=136)$ & $7(5)$ \\
$3-6$ mm $(n=306)$ & $28(9)$ \\
$7-10 \mathrm{~mm}(n=129)$ & $23(17)$ \\
$11-25 \mathrm{~mm}(n=59)$ & $18(31)$ \\
$>25 \mathrm{~mm}(n=4)$ & $1(25)$ \\
Residual of coiled aneurysm $(n=32)$ & $2(6)$
\end{tabular}

$A C A$, anterior cerebral artery; $I C A$, interior carotid artery; $M C A$, middle cerebral artery; $P C$, posterior circulation

based residuals. The mean FU was 31 months (SD: \pm 23.3 , range 2.3-93 months).

The reasons for missing FU differed. In 12 cases, FU angiography was recommended but the patient did not attend the FU examination. In 7 cases, the patients were in poor neurological condition and FU angiography was recommended only if the patients improved during their postoperative courses. These were all patients who had suffered a severe SAH. In 4 dog-ear cases, FU angiography was not recommended

Table 4 Factors associated with postoperative aneurysm residuals. The factor positively associated with postoperative aneurysm residuals is aneurysm size $11-25 \mathrm{~mm}$. The location of the "MCA" aneurysm is associated with a reduced risk for postoperative aneurysm residuals. (Binary logistic regression)

\begin{tabular}{lll}
\hline Parameter & OR $(95 \% \mathrm{CI})$ & $p$ \\
\hline Aneurysm location & & \\
ACA & $0.67(0.24-1.84)$ & 0.44 \\
ICA & $1.35(0.51-3.61)$ & 0.55 \\
MCA & $0.31(0.12-0.83)$ & 0.02 \\
Aneurysm size $n(\%)$ & & \\
$<3 \mathrm{~mm}$ & $0.98(0.19-4.99)$ & 0.98 \\
$3-6 \mathrm{~mm}$ & $2.04(0.46-9.12)$ & 0.35 \\
$7-10 \mathrm{~mm}$ & $4.23(0.95-20.60)$ & 0.06 \\
$11-25 \mathrm{~mm}$ & $9.36(1.96-44.61)$ & 0.005 \\
$>25 \mathrm{~mm}$ & $4.46(0.37-66.74)$ & 0.28 \\
Surgery in acute phase & $0.67(0.38-1.16)$ & 0.16 \\
\hline
\end{tabular}

$A C A$, anterior cerebral artery; $I C A$, interior carotid artery; $M C A$, middle cerebral artery
78 residuals

62 dog ears

16 broad based

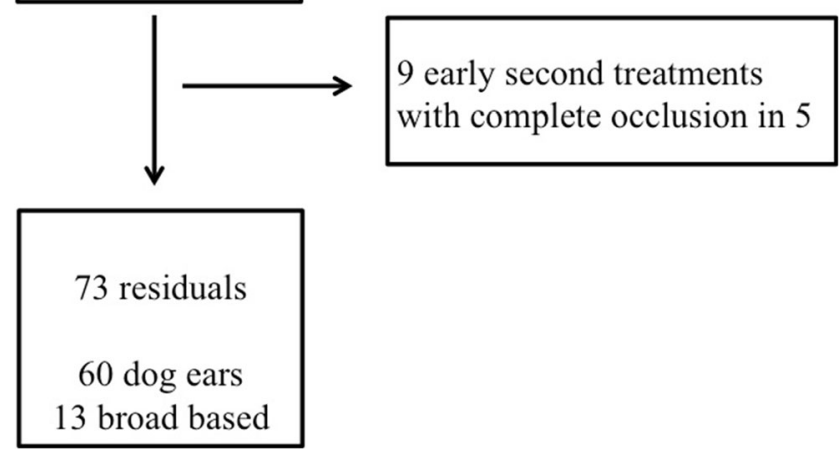

Fig. 2 Flowchart of aneurysm residuals

because of the small size of the aneurysm remnant. In 9 cases, a FU recommendation was not specified at discharge.

Follow-up of dog-ear remnants and broad-based remnants

Table 5 shows the course of dog-ear residuals during FU. The major part of the dog-ear residuals stayed stable, decreased in size, or closed during FU (94\%). An increase in size was recorded in two cases.

During FU, two of the broad-based aneurysms increased in size, one decreased, and none closed spontaneously. The number of broad-based aneurysms in FU was small $(n=7)$ (Table 6). Figures 3 and 4 show typical DSA findings of a dog ear and a broad-based aneurysm residual.

\section{Treatment of aneurysm residuals during follow-up}

All aneurysm remnants with increasing size were treated ( 2 in the dog-ear group, 2 in the broad-based group). The 2 progressive dog-ear residuals were treated 1 year after clipping by coiling and stent-assisted coiling. The progressive broadbased residuals were treated via clipping in one case at 6 months after the first surgery and by coiling in the other case at 1 year after surgery.

Two broad-based aneurysm residuals were treated irrespective of the residual growth during the FU. In 1 case, the broadbased residual of a paraophthalmic aneurysm was treated with a flow diverter 6 months postoperatively. In another case, a paraophthalmic ICA aneurysm was clipped and postoperative angiography revealed a small remnant. Early second surgery was performed, but a postoperative DSA still showed an aneurysm residual. One year later, the aneurysm residual was treated with stent-assisted coiling and showed no further growth during FU. 
Table 5 Behavior of dog-ear residuals in follow-up $(n=33)$

\begin{tabular}{lllll}
\hline & $\begin{array}{l}\text { Stable size in } \\
\text { FU }\end{array}$ & $\begin{array}{l}\text { Decreased size in } \\
\text { FU }\end{array}$ & $\begin{array}{l}\text { Closed in } \\
\text { FU }\end{array}$ & $\begin{array}{l}\text { Increased in } \\
\text { size }\end{array}$ \\
\hline $\begin{array}{l}\text { Number of aneurysm residuals } n, \\
(\%)\end{array}$ & $22(67)$ & $3(9)$ & $6(18)$ & $2(6)$ \\
\hline
\end{tabular}

\section{Discussion}

In the current study, we analyzed the risk factors for aneurysm remnants after microsurgical clipping and the course of aneurysm remnants in FU. Postoperative angiography revealed a rate of $12 \%$ for residual aneurysm filling in clipped aneurysms in our series. These data are consistent with other published studies, as the frequency of residual filling of aneurysms after clipping is reported to range from 2.3 to $19.3 \%$. One series from Korea revealed an incidence of residual filling of even $49 \%$ when controlled with 3D angiography and defining a remnant size from $1 \mathrm{~mm}$ onward $[1-5,7,9,12,13,15,20$, $24,25,27,28,31,32]$. Our series confirms the need for early postoperative angiography to certify the success of the surgery and, in the case of remnants, to ensure that FU or further therapy can be scheduled appropriately. The necessity for postoperative angiography has been discussed previously [8, $10,12]$, and this procedure remains the absolute standard for control after aneurysm surgery.

\section{Risk factors for incomplete aneurysm occlusion}

A greater aneurysm size $(11-25 \mathrm{~mm})$ was associated with incomplete surgical occlusion. Jabbarli et al. described similar results for aneurysms sized $>12 \mathrm{~mm}$ and an association with a higher risk for a clip remnant [18].

The location of the aneurysm is considered important for the occurrence of an aneurysm remnant, as the risk of aneurysm remnants seem to be greater for paraophthalmic, anterior communicating artery, and basilar artery aneurysms [18, 27, 32]. Our series revealed a lower risk for aneurysm residuals following clipping of MCA aneurysms, in agreement with previously described findings and reflecting the technically easier accessibility as compared to paraophthalmic and posterior circulation aneurysms $[21,28]$.

Direct intraoperative catheter angiography within a hybrid operating room is described as potentially beneficial for the occlusion rates of the aneurysms $[22,26]$. However, in our series, only 5 patients underwent early clip revision surgery. This was primarily due to the technically difficult configuration of the aneurysm; therefore, it could not be avoided with the use of intraoperative DSA.

Angiographic and clinical follow-up of aneurysm remnants

In our series, the major part of dog-ear residuals remained stable, decreased in size, or vanished during the FU. Therefore, foregoing angiographic controls might be justified, particularly in patients without risk factors such as a previous history of SAH, hereditary or de novo aneurysms, hypertonus, or smoking. A younger age should also be regarded as a potential risk factor because of the previously reported increased risk of regrowth of aneurysm residuals in patients under 45 years old [18].

In 2004, Akyüz et al. performed a long-term angiographic FU (median 46.6 months) of 166 clipped aneurysms, with 7 residuals $(4.2 \%)$, using the Sindou residual grading system. They reported 5 small neck residuals and 2 with a broader residual. One case of regrowth was recorded in a small remnant and led to reoperation, but no cases of regrowth occurred among the larger remnants. These surgeons also had one case of spontaneous thrombosis of a small remnant but experienced no cases of rebleeding during FU. They concluded that small aneurysm remnants may stay stable [3].

The FU of broad-based aneurysms in our series was incomplete, but it revealed a high rate of regrowth $(28 \%)$. This was also observed by David et al., who noted aneurysm regrowth in 3 out of 4 cases [9]. Only one broad-based residual decreased in size during FU, but it did not close completely. Broad-based residuals seem to have a high risk of regrowth and should be scheduled for FU, as the need for retreatment seems to occur frequently. Furthermore, the CARAT study revealed a size-dependent risk of rebleeding in clipped and coiled aneurysms [19].

In treated aneurysms after $\mathrm{SAH}$, the risk of rehemorrhage has been estimated as $0.11-0.21 \%$ in the first year for coiled aneurysms and $0.0-0.03 \%$ for clipped aneurysms [10, 19]. In our series, no case of rebleeding occurred in the incompletely clipped aneurysms within the FU of a maximum of 93 months. David et al. estimated the risk of rebleeding in aneurysms with postoperative remnants as $1.5 \%$ per year [9]. A series of 715 surgically treated patients reported by Feuerberg
Table 6 Behavior of broad-based residuals in follow-up $(n=7)$

\begin{tabular}{llll}
\hline & Stable size in FU & Decreased size in FU & Increased in size \\
\hline Number of aneurysm residuals $n,(\%)$ & $4(60)$ & $1(14)$ & $2(28)$ \\
\hline
\end{tabular}


Fig. 3 a Preoperative DSA demonstrating an incidental paraophthalmic ICA aneurysm which was scheduled for clipping; b postoperative DSA reveals small dog-ear residual. This patient was scheduled for follow-up DSA, which showed a stable result
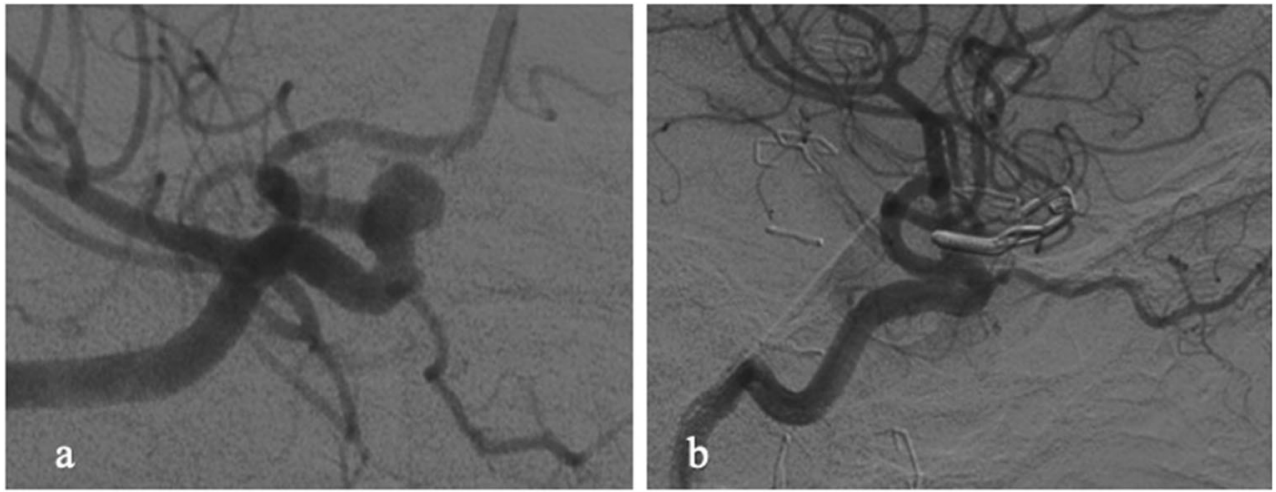

et al. revealed a risk of rebleeding in aneurysm residuals of $0.38-0.79 \%$ per year, leading to a discussion of whether this risk of rebleeding might justify the risk of reoperation [15]. Rauzzino et al. determined a relatively high risk of rebleeding in aneurysm remnants. Surgery in 312 aneurysms led to a remnant rate of $4.2 \%(n=13)$. Only 4 of these remnants were not treated immediately after initial surgery. All 4 of these remaining aneurysm remnants became symptomatic within 2 years: 3 with a new SAH and 1 because of a local mass effect [28]. Configuration of the remnants was not further specified in this study.

Furthermore, radiation exposure, periprocedural complications, and costs should also be factored into the decision for invasive angiographic controls. The development of less invasive but adequate imaging procedures with lower radiation exposure for the patient, such as metal artifact reduced MRI, would be desirable. However, even the most recent, newly developed MRI sequences are not able to suppress the artifacts so strongly to be able to determine the fine differences in size or configuration of the neck remnant [16]. The MRI is nowadays much more useful in checking untreated aneurysms or in excluding de novo formations in predisposed patients.

$\mathrm{CT}$ angiography (CT-A) is less invasive and has been compared to classical DSA in different studies. The sensitivity for detection of postoperative aneurysm remnants varied between
50-100\% comparing CT-A to DSA $[6,11,14,23,30,33,34]$. The precision of the CT-A strongly decreases with the usage of multiple clips, in cases with posterior circulation aneurysms and in small remnants.

Sagara et al. came to the conclusion that the usage of multiple clips is an indication for 3D DSA [30]. Bharatha et al. reported a sensitivity of $88 \%$ for the detection of aneurysm remnants after clipping when comparing DSA to CT-A, whereas the sensitivity for a small neck remnant (mean size $1 \mathrm{~mm}$ ) was only $20 \%$ [6].

Dundar et al. compared traditional DSA to subtraction CT-A and came to the conclusion that DSA remains the gold standard as residuals $<3 \mathrm{~mm}$ are not reliably examined with CT-A [14].

In our opinion and according to previous publications, CT-A might be suitable, for long-term follow-up controls when DSA controls were initially stable and CT-A quality is good [17].

\section{Study limitations}

One limitation of our study is its retrospective data collection. Furthermore, FU is not available for almost half the patients with incompletely occluded aneurysms, either due to the poor clinical condition of certain SAH patients or due to the estimated low-risk factors based on patient's postoperative angiography and medical history. This may obscure the true data
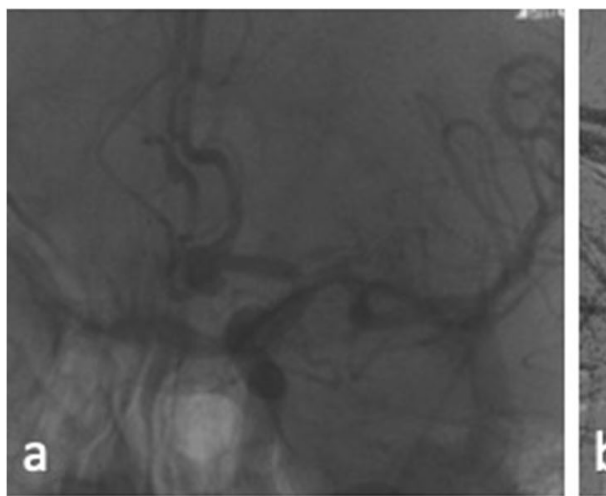

Fig. 4 a Preoperative DSA of an Acoma aneurysm in a patient with SAH H\&H II; b postoperative DSA 1 showed a broad-based aneurysm residual and the patient underwent revision surgery on the same day; $\mathbf{c}$ postoperative DSA 2 demonstrates now complete occlusion of the residual, which was achieved by placement of further clips 
concerning growth dynamics of incompletely occluded aneurysms.

\section{Conclusions}

MCA aneurysms appear to have a lower risk for postoperative aneurysm residuals than is observed for an aneurysm in other locations. A large part of dog-ear residuals appear to remain stable, decrease in size, or close during FU. Management of aneurysm remnants should consider individual risk factors and the remnant configuration.

Funding Open Access funding enabled and organized by Projekt DEAL.

\section{Compliance with ethical standards}

Conflict of interest The authors declare that they have no conflict of interest.

Ethical approval All procedures performed in studies involving human participants were in accordance with the ethical standards of the institutional and/or national research committee and with the 1964 Helsinki declaration and its later amendments or comparable ethical standards.

Open Access This article is licensed under a Creative Commons Attribution 4.0 International License, which permits use, sharing, adaptation, distribution and reproduction in any medium or format, as long as you give appropriate credit to the original author(s) and the source, provide a link to the Creative Commons licence, and indicate if changes were made. The images or other third party material in this article are included in the article's Creative Commons licence, unless indicated otherwise in a credit line to the material. If material is not included in the article's Creative Commons licence and your intended use is not permitted by statutory regulation or exceeds the permitted use, you will need to obtain permission directly from the copyright holder. To view a copy of this licence, visit http://creativecommons.org/licenses/by/4.0/.

\section{References}

1. Acevedo JC, Turjman F, Sindou M (1997) Postoperative arteriography in surgery for intracranial aneurysm. Prospective study in a consecutive series of 267 operated aneurysms. Neuro-Chirurgie 43: 275-284

2. Ahn SS, Kim YD (2010) Three-dimensional digital subtraction angiographic evaluation of aneurysm remnants after clip placement. J Korean Neurosurg Soc 47:185-190. https://doi.org/10.3340/jkns. 2010.47.3.185

3. Akyuz M, Tuncer R, Yilmaz S, Sindel T (2004) Angiographic follow-up after surgical treatment of intracranial aneurysms. Acta Neurochir 146:245-250; discussion 250. https://doi.org/10.1007/ s00701-003-0206-z

4. Allcock JM, Drake CG (1963) Postoperative angiography in cases of ruptured intracranial aneurysm. J Neurosurg 20:752-759. https:// doi.org/10.3171/jns.1963.20.9.0752

5. Asgari S, Doerfler A, Wanke I, Schoch B, Forsting M, Stolke D (2002) Complementary management of partially occluded aneurysms by using surgical or endovascular therapy. J Neurosurg 97:843-850. https://doi.org/10.3171/jns.2002.97.4. 0843

6. Bharatha A, Yeung R, Durant D, Fox AJ, Aviv RI, Howard P, Thompson AL, Bartlett ES, Symons SP (2010) Comparison of computed tomography angiography with digital subtraction angiography in the assessment of clipped intracranial aneurysms. J Comput Assist Tomogr 34:440-445. https://doi.org/10.1097/RCT. 0b013e3181d27393

7. Choi SW, Ahn JS, Park JC, Kwon do H, Kwun BD, Kim CJ (2012) Surgical treatment of unruptured intracranial middle cerebral artery aneurysms: angiographic and clinical outcomes in 143 aneurysms. J Cerebrovasc Endovasc Neurosurg 14:289-294. https://doi.org/10. 7461/jcen.2012.14.4.289

8. Cronk K, Spetzler RF (2010) Commentary for recurrent intracranial aneurysms after successful neck clipping. World Neurosurg 74: 437-438. https://doi.org/10.1016/j.wneu.2010.10.032

9. David CA, Vishteh AG, Spetzler RF, Lemole M, Lawton MT, Partovi S (1999) Late angiographic follow-up review of surgically treated aneurysms. J Neurosurg 91:396-401. https://doi.org/10. 3171/jns.1999.91.3.0396

10. de Sousa AA (2010) Follow-up of cerebral aneurysms after neck clipping. World Neurosurg 74:435-436. https://doi.org/10.1016/j. wneu.2010.08.003

11. Dehdashti AR, Binaghi S, Uske A, Regli L (2006) Comparison of multislice computerized tomography angiography and digital subtraction angiography in the postoperative evaluation of patients with clipped aneurysms. J Neurosurg 104:395-403. https://doi. org/10.3171/jns.2006.104.3.395

12. Drake CG, Allcock JM (1973) Postoperative angiography and the "slipped" clip. J Neurosurg 39:683-689. https://doi.org/10.3171/ jns.1973.39.6.0683

13. Drake CG, Friedman AH, Peerless SJ (1984) Failed aneurysm surgery. Reoperation in 115 cases. J Neurosurg 61:848-856. https:// doi.org/10.3171/jns.1984.61.5.0848

14. Dundar TT, Aralasmak A, Kitis S, Yılmaz FT, Abdallah A (2019) Comparison of subtracted computed tomography from computed tomography perfusion and digital subtraction angiography in residue evaluation of treated intracranial aneurysms. World Neurosurg 132:e746-e751. https://doi.org/10.1016/j.wneu.2019.08.028

15. Feuerberg I, Lindquist C, Lindqvist M, Steiner L (1987) Natural history of postoperative aneurysm rests. J Neurosurg 66:30-34. https://doi.org/10.3171/jns.1987.66.1.0030

16. Friedrich B, Wostrack M, Ringel F, Ryang YM, Forschler A, Waldt S, Zimmer C, Nittka M, Preibisch C (2016) Novel metal artifact reduction techniques with use of slice-encoding metal artifact correction and view-angle tilting $\mathrm{mr}$ imaging for improved visualization of brain tissue near intracranial aneurysm clips. Clin Neuroradiol 26:31-37. https://doi.org/10.1007/s00062-014-0324-4

17. Gölitz P, Struffert T, Ganslandt O, Saake M, Lücking H, Rösch J, Knossalla F, Doerfler A (2012) Optimized angiographic computed tomography with intravenous contrast injection: an alternative to conventional angiography in the follow-up of clipped aneurysms? J Neurosurg 117:29-36. https://doi.org/10.3171/2012.3.Jns111895

18. Jabbarli R, Pierscianek D, Wrede K, Dammann P, Schlamann M, Forsting M, Muller O, Sure U (2016) Aneurysm remnant after clipping: the risks and consequences. J Neurosurg 125(5):1249 1255. https://doi.org/10.3171/2015.10.jns151536

19. Johnston SC, Dowd CF, Higashida RT, Lawton MT, Duckwiler GR, Gress DR, Investigators C (2008) Predictors of rehemorrhage after treatment of ruptured intracranial aneurysms: the Cerebral Aneurysm Rerupture After Treatment (CARAT) study. Stroke 39: 120-125. https://doi.org/10.1161/STROKEAHA.107.495747

20. Kang HS, Han MH, Kwon BJ, Jung SI, Oh CW, Han DH, Chang KH (2004) Postoperative 3D angiography in intracranial aneurysms. AJNR Am J Neuroradiol 25:1463-1469 
21. Kivisaari RP, Porras M, Ohman J, Siironen J, Ishii K, Hernesniemi J (2004) Routine cerebral angiography after surgery for saccular aneurysms: is it worth it? Neurosurgery 55:1015-1024

22. Klopfenstein JD, Spetzler RF, Kim LJ, Feiz-Erfan I, Han PP, Zabramski JM, Porter RW, Albuquerque FC, McDougall CG, Fiorella DJ (2004) Comparison of routine and selective use of intraoperative angiography during aneurysm surgery: a prospective assessment. J Neurosurg 100:230-235. https://doi.org/10.3171/jns. 2004.100.2.0230

23. Kunert P, Prokopienko M, Gola M, Dziedzic T, Jaworski M, Marchel A (2013) Assessment of long-term results of intracranial aneurysm clipping by means of computed tomography angiography. Neurol Neurochir Pol 47:18-26. https://doi.org/10.5114/ninp. 2012.31549

24. Le Roux PD, Elliott JP, Eskridge JM, Cohen W, Winn HR (1998) Risks and benefits of diagnostic angiography after aneurysm surgery: a retrospective analysis of 597 studies. Neurosurgery 42: 1248-1254 discussion 1254-1245

25. Macdonald RL, Wallace MC, Kestle JR (1993) Role of angiography following aneurysm surgery. J Neurosurg 79:826-832. https:// doi.org/10.3171/jns.1993.79.6.0826

26. Marbacher S, Mendelowitsch I, Gruter BE, Diepers M, Remonda L, Fandino J (2018) Comparison of 3D intraoperative digital subtraction angiography and intraoperative indocyanine green video angiography during intracranial aneurysm surgery. J Neurosurg 131(1): 64-71. https://doi.org/10.3171/2018.1.jns172253

27. Meyer B, Urbach H, Schaller C, Baslam M, Nordblom J, Schramm J (2004) Immediate postoperative angiography after aneurysm clipping-implications for quality control and guidance of further management. Zentralbl Neurochir 65:49-56. https://doi.org/10. $1055 / \mathrm{s}-2004-816267$
28. Rauzzino MJ, Quinn CM, Fisher WS 3rd (1998) Angiography after aneurysm surgery: indications for "selective" angiography. Surg Neurol 49:32-40 discussion 40-31

29. Raymond J, Roy D, Bojanowski M, Moumdjian R, L'Espérance G (1997) Endovascular treatment of acutely ruptured and unruptured aneurysms of the basilar bifurcation. J Neurosurg 86:211-219. https://doi.org/10.3171/jns.1997.86.2.0211

30. Sagara Y, Kiyosue H, Hori Y, Sainoo M, Nagatomi H, Mori H (2005) Limitations of three-dimensional reconstructed computerized tomography angiography after clip placement for intracranial aneurysms. J Neurosurg 103:656-661. https://doi.org/10.3171/jns. 2005.103.4.0656

31. Sato S, Suzuki J (1971) Prognosis in cases of intracranial aneurysm after incomplete direct operations. Acta Neurochir 24:245-252

32. Sindou M, Acevedo JC, Turjman F (1998) Aneurysmal remnants after microsurgical clipping: classification and results from a prospective angiographic study (in a consecutive series of 305 operated intracranial aneurysms). Acta Neurochir 140:1153-1159

33. Teksam M, McKinney A, Casey S, Asis M, Kieffer S, Truwit CL (2004) Multi-section CT angiography for detection of cerebral aneurysms. AJNR Am J Neuroradiol 25:1485-1492

34. van Loon JJ, Yousry TA, Fink U, Seelos KC, Reulen HJ, Steiger HJ (1997) Postoperative spiral computed tomography and magnetic resonance angiography after aneurysm clipping with titanium clips. Neurosurgery 41:851-856; discussion 856-857. https://doi.org/10. 1097/00006123-199710000-00016

Publisher's note Springer Nature remains neutral with regard to jurisdictional claims in published maps and institutional affiliations. 